Xujian Lyu

Haiwen $T u$

De Xie

Jianglong Sun

http://dx.doi.org/10.21278/brod69101

\title{
ON RESISTANCE REDUCTION OF A HULL BY TRIM OPTIMIZATION
}

UDC 629.5.012.442:629.5.015.2

Original scientific paper

\begin{abstract}
The paper aims at conducting trim optimization for a hull to reveal the influence of trim on wave resistance by a potential-based panel method coupled with a response surface method. First, a numerical program for solving the linear free-surface flow problem of a hull moving with a uniform speed in calm water is built by the panel method. The S60 hull model is used to validate the numerical procedure. Next, calculation for hull is performed with two different trims at a wide range of Froude number; resistance test is conducted to validate the numerical method in demonstrating the influence of trim on wave resistance. Finally, a response surface of wave resistance is constructed with respect to variations of trim and Froude number, using the database of wave resistance calculated by the surface method. In this way, a framework is developed to perform trim optimization. The optimum trim point for the present hull shows a significant improvement in both wave resistance and total resistance, compared with that of even keel and the worst trim point. The optimization framework is proved to be effective in energy saving due to resistance reduction.
\end{abstract}

Key words: $\quad$ Trim optimization; Wave resistance; Panel method; Response surface

\section{Introduction}

In the face of global climate change, the importance of addressing greenhouse gas emissions attracts worldwide attention gradually. For shipping industry, the problems of greenhouse gas emission and fuel efficiency in operation are becoming more and more important due to the requirement of Environmental Ship Index (ESI) from International Maritime Organization (IMO) [1, 2]. When it comes to green shipping, one of the main concerns about ship hydrodynamics is resistance reduction. As it is well known, employing excellent hull forms or optimizing hull lines with low resistance in ship design stage is an effective measure for this goal [3-6]. However, it usually requires modifications to hull lines, which may cost lots of money and time for ships in operation. Another option to optimize the resistance performance without modifications to hull is increasing the operational efficiency of ships [7]. Traditionally, ships have been optimized for minimum resistance at the designing trim condition, which is the even keel trim condition or trimming somewhat aft for most 
ships. Thus, one might think that the optimum trim condition, when wave-making resistance is considered, should not always be the designing one. With typical wave-making resistance taken into account, the paper is therefore to explore the optimum trim condition for a certain hull at given Froude numbers and displacement.

As one of the most widely used tools for wave-making calculation in hydrodynamics, the potential-based panel method deals with the Laplace boundary-value problem with solving integral equation only at boundaries of the flow field [8-11]. Therefore, it is usually more effective in wave resistance calculation than other tools such as viscous flow based Computational Fluid Dynamics (CFD). Ghassemi and Kohansal studied the generation of wave due to moving hydrofoil in steady streams close to free surface, employing a potential based panel method [12]. A modified Rankine panel method was used to improve the prediction of wave pattern and wave-making resistance for ships of full form by Peng et al. [13], and an alternative method was first developed to satisfy the free surface boundary conditions on arbitrary body-fitted lines instead of streamlines. Lv et al. employed a potentialbased boundary element method to determine a better trim condition with lower wave resistance of the hull. However, without an optimization model, the trim had not been proved to be optimum for the hull. Iakovatos et al. [14] experimentally studied the impact of trim angles on resistance. In addition, Sherbaz and Duan assessed the influence of trim on ship resistance with CFD as well [15]. More recently, Sun et al. [16] developed a response surface based program with database from experiment and CFD to perform trim optimization. The optimized results had been validated by real ship test as well. However, experiment and CFD require a lot of money or computer resource input to assess the influence of trim on ship resistance.

In this study, an efficient and economical framework is developed, to get resistance reduction based on trim optimization for a certain hull. Other than using a double model based boundary element method for wave resistance calculation [17], a direct potential-based panel method is applied to build a database for wave resistance. The Series 60 hull is utilized as a verification example and model test for a certain hull is conducted to verify the influence of trim on wave resistance as well. Finally, the response surface method, with surface fitting by Lagrange interpolation function instead of polynomial function, is applied to perform trim optimization, and the influence of trim on wave resistance and total resistance is evaluated.

\section{Statement of the Boundary Value Problem}

Let us consider a ship moving with a constant speed $U$ in calm water. A ship-fixed Cartesian coordinate system $O-x y z$ is defined with origin $O$ located at the mid-ship on the calm water surface and the $x$-axis positive towards the ship stern. The $y$-axis positive extends to starboards and the $z$-axis is vertically towards (Fig. 1).

It is assumed that the fluid is incompressible and inviscid, and the flow is irrotational. The total velocity potential $\Phi$ can be expressed as a sum of the oncoming velocity potential $\phi_{\infty}$ and the perturbation potential $\phi$, namely

$$
\Phi=\phi_{\infty}+\phi=\vec{U} \cdot \vec{x}+\phi
$$

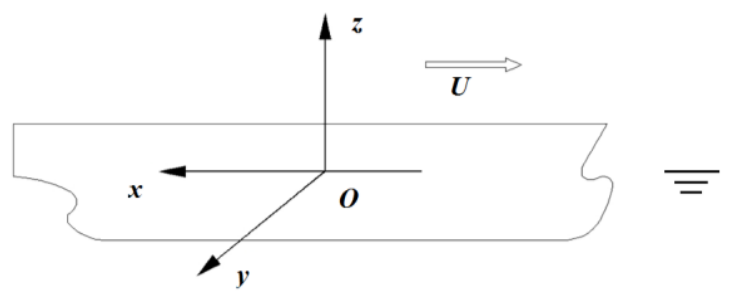

Fig. 1 Definition sketch of the coordinate system 
Conservation of the mass applied to the potential flow gives the Laplace equation as a governing equation

$$
\nabla^{2} \phi=0
$$

Then motion of the flow can be uniquely defined by imposing the boundary condition on the boundary surface as follows:

1) Hull boundary condition

$$
\frac{\partial \phi}{\partial n}=-\vec{U} \cdot \vec{n} \text { on } S_{H}
$$

where $\vec{n}$ is the unit vector normal to the boundary, defined positive when pointing into the fluid region.

2) Linearized free surface boundary condition can be simplified as

$$
\frac{\partial \phi}{\partial z}+\frac{U^{2}}{g} \frac{\partial^{2} \phi}{\partial x^{2}}=0 \text { on } S_{F}
$$

where $g$ is gravity acceleration.

In addition, a four-point finite upstream scheme is used to ensure that the free-surface waves vanish upstream of the disturbance.

By applying Green's theorem, the equation about the perturbation potential can be expressed as

$$
\int_{S_{H}+S_{F}}\left\{\phi \frac{\partial G}{\partial n}-G \frac{\partial \phi}{\partial n}\right\} \mathrm{d} S=0
$$

For the problem of flow around the hull. Equation (4) can be written as

$$
4 \pi E \phi(p)=\int_{S_{H}} \phi(q) \frac{\partial G}{\partial n_{q}} \mathrm{~d} S-\int_{S_{H}} G \frac{\partial \phi(q)}{\partial n_{q}} \mathrm{~d} S-\int_{S_{F}} \phi(q) \frac{\partial G}{\partial n_{q}} \mathrm{~d} S
$$

where

$$
E= \begin{cases}\frac{1}{2} & \text { on } S_{H} \\ 1 & \text { on } S_{F}\end{cases}
$$

Green's function $G$ is given by

$$
G=\frac{1}{R(p, q)}+\frac{1}{R^{\prime}(p, q)}
$$

where $R=\sqrt{(x-\xi)^{2}+(y-\eta)^{2}+(z-\zeta)^{2}}$ is the distance between field point and the source point, and $R^{\prime}=\sqrt{(x-\xi)^{2}+(y-\eta)^{2}+(z+\zeta)^{2}}$ is the distance between field point and the image of source point.

The hull surface $S_{H}$ and the free surface $S_{F}$ are discretized into a number of $N_{H}$ and $N_{F}$ panels respectively to get an approximate solution of the problem [18]. The perturbation potential $\phi$ and its normal derivative $\frac{\partial \phi}{\partial n}$ are assumed constant at each panel and equation about $\phi$ and $\frac{\partial \phi}{\partial n}$ can be obtained as 


$$
\phi(p)=\sum_{j=1}^{N_{H}}\left\{\phi(q) \cdot I_{H D}+\frac{\partial \phi(q)}{\partial n_{q}} \cdot I_{H S}\right\}+\sum_{j=1}^{N_{F}} \frac{\partial \phi(q)}{\partial n_{q}} \cdot I_{F S}
$$

where $I_{H D}, I_{H S}$ and $I_{F S}$ are influence coefficients proposed by Morino et al. [19] and the details can be referred to the literature [20].

As the free surface should be satisfied on the true surface, an iterative method is used to obtain the solution of equation (9). To get acceptable results, the iteration number is about $4 \sim 7$ in this paper.

The wave resistance coefficient

$$
C_{w}=\frac{R_{w}}{(1 / 2) \rho U^{2} S_{W}}=\frac{1}{S_{W}} \sum_{i=1}^{N_{H} / 2} C_{p}(i) n_{x i} \Delta S_{W i}
$$

where $S_{W}$ is the wetted surface area of the hull in calm water, $\rho$ is the water density, $\Delta S_{W i}$ is the area of the panel element, $n_{x i}$ is $x$-component of the unit vector normal to the surface panel element, and $C_{p}$ is the pressure coefficient determined by the Bernoulli's equation.

The wave height can be calculated with the linearized kinematic free surface condition

$$
\varsigma(x, y)=-\frac{U}{g} \frac{\partial \phi}{\partial x} \quad \text { on } z=0
$$

\section{Sample calculation and validation}

The Series 60 hull model $\left(C_{b}=0.6\right)$ is used as an example to check the reliability for the numerical procedure of present panel method [21]. The free surface is discretized $\left(-1 \leq x / L_{\mathrm{pp}}\right.$ $\left.\leq 2,-1 \leq y / L_{\mathrm{pp}} \leq 1\right)$ by $100 \times 40$ quadrilateral panels and the hull by 1346 panels, $5 \times 6$ for the stern and $28 \times 47$ for other parts of the hull respectively. Calculation is conducted for the S60 hull with fixed trim and sinkage and computed wave resistance coefficient is compared with that obtained from the literature by Tarafder \& Suzuki [18], as shown in Fig. 2. Though there are some differences between the results of wave resistance from present study and that from literature at some Froude numbers, the overall agreement of the results is quite acceptable. In addition, numerical results on wave profiles along the body surface at $\mathrm{Fn}=0.22$ and $\mathrm{Fn}=0.34$ are shown in Figs 3 and 4 respectively. The variation trend of wave profiles from present study and literature matches well with each other. The present results show higher accuracy than that from literature when compared with experimental results, as shown in the figures. The comparisons show that the present numerical method is able to catch typical flow characters around the hull, and thus it can be concluded that the numerical procedure is feasible for wave resistance and wave profile prediction. Subsequently, the numerical method is applied for the calculation of a HUST (Huazhong University of Science $\&$ Technology) hull [17], of which the main parameters are summarized in table 1. Both the hull surface and the free surface domain $\left(-1 \leq x / L_{\mathrm{pp}} \leq 2,-1 \leq y / L_{\mathrm{pp}} \leq 1\right)$ are discretized by quadrilateral panels, as shown in Figs 5 and 6 . The displacement of the hull is kept constant during the calculation.

To provide further validation of the numerical procedure, resistance tests of the HUST hull were performed at the towing tank laboratory of the School of Naval Architecture and Ocean Engineering, Huazhong University of Science and Technology, Wuhan, P. R. China. The principle dimension of the towing tank is $175 \times 6 \times 4 \mathrm{~m}$ [16]. According to the ITTC guidelines, the wave resistance coefficient of HUST hull from model test can be written as

$$
C_{t}=(1+k) \cdot C_{f}+\mathrm{C}_{w}
$$




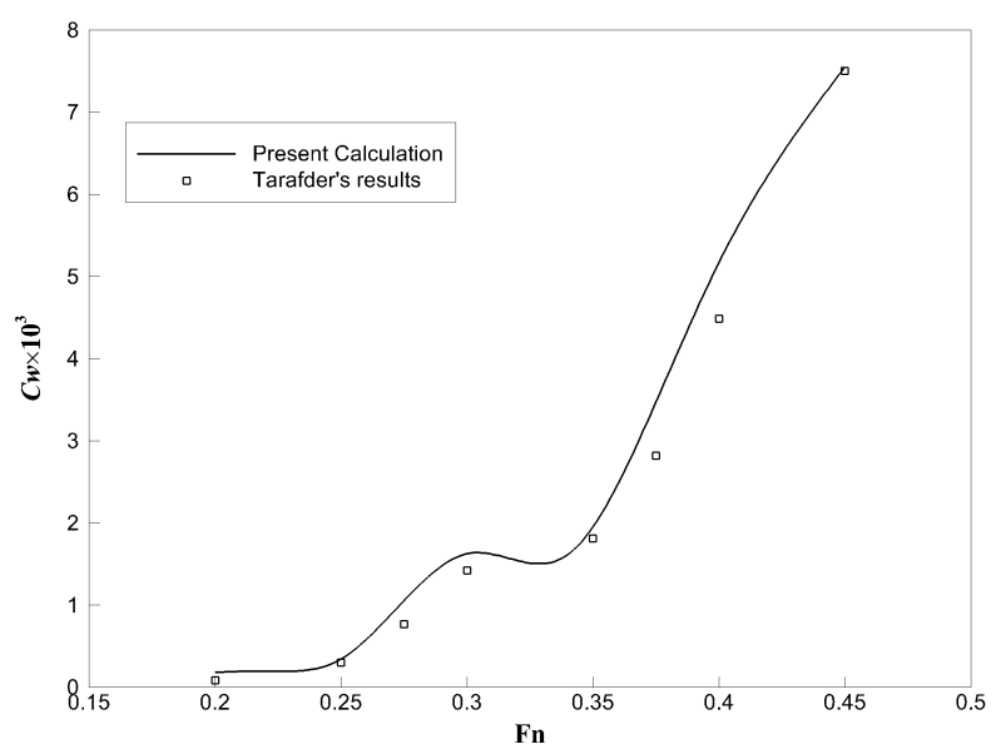

Fig. 2 Wave resistance of S60 hull

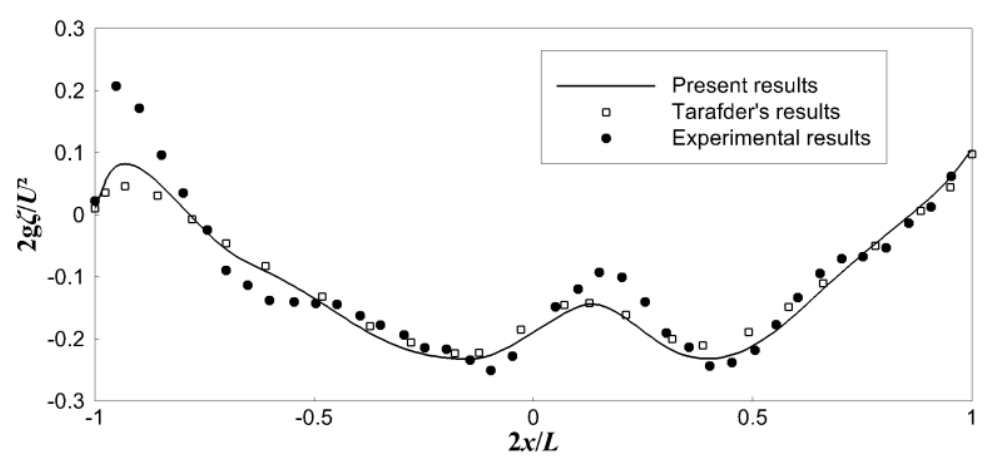

Fig. 3 Wave profiles of the $\mathrm{S} 60$ hull at $\mathrm{Fn}=0.22$

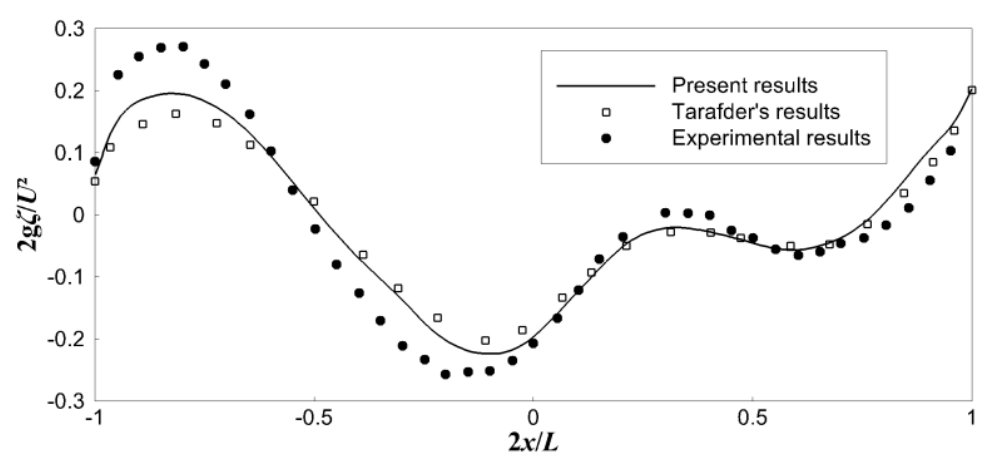

Fig. 4 Wave profiles of the S60 hull at Fn $=0.34$.

where $C_{t}$ is the total resistance coefficient got from model test, $C_{f}$ is the frictional resistance coefficient determined by the ITTC ' 57 correlation line and $k$ is the form factor determined by Prohaska method adopted by ITTC in 1978.

The trim condition of present hull is defined by the aft draft $T_{a}$ and the fore draft $T_{f}$, that is

$$
\operatorname{Trim}=T_{f}-T_{a}
$$

where Trim $>0$ indicates trim by bow and Trim $<0$ indicates trim by stern, as shown in Fig. 7. 
De Xie, Jianglong Sun

Table 1 Typical panel arrangement for the hull

\begin{tabular}{|c|c|c|}
\hline Descriptions & Parameters & Value \\
\hline Volume of displacement & $\nabla(\mathrm{m} 3)$ & 0.7136 \\
\hline Wetted surface area & $S_{\mathrm{w}}(\mathrm{m} 2)$ & 4.4961 \\
\hline Length between perpendiculars & $L_{\mathrm{PP}}(\mathrm{m})$ & 4.4225 \\
\hline Beam & $B(\mathrm{~m})$ & 0.6950 \\
\hline Draft & $D(\mathrm{~m})$ & 0.2687 \\
\hline Block coefficient & $C_{\mathrm{B}}$ & 0.7443 \\
\hline & & \\
\hline
\end{tabular}

Fig. 5 Typical panel arrangement for the HUST hull

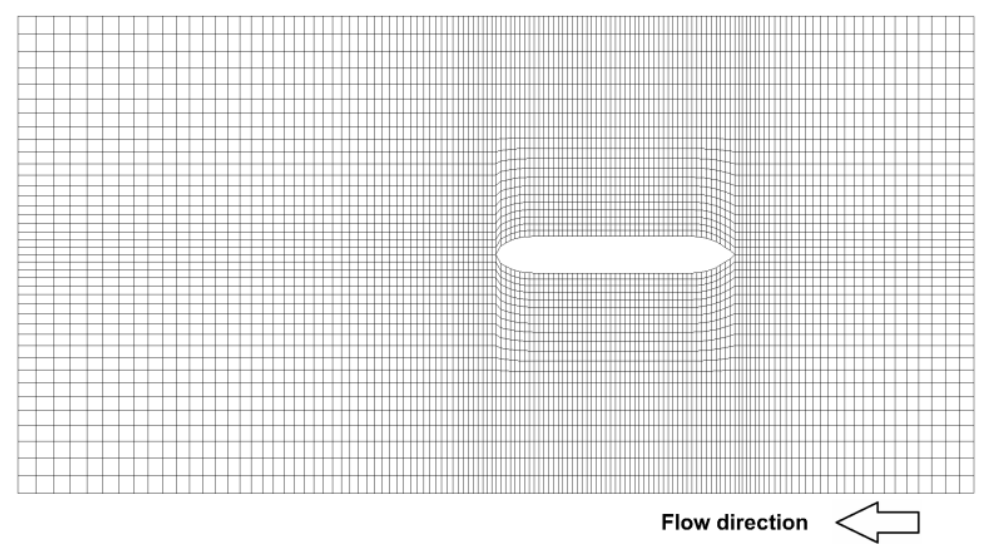

Fig. 6 Typical panel arrangement for the free surface
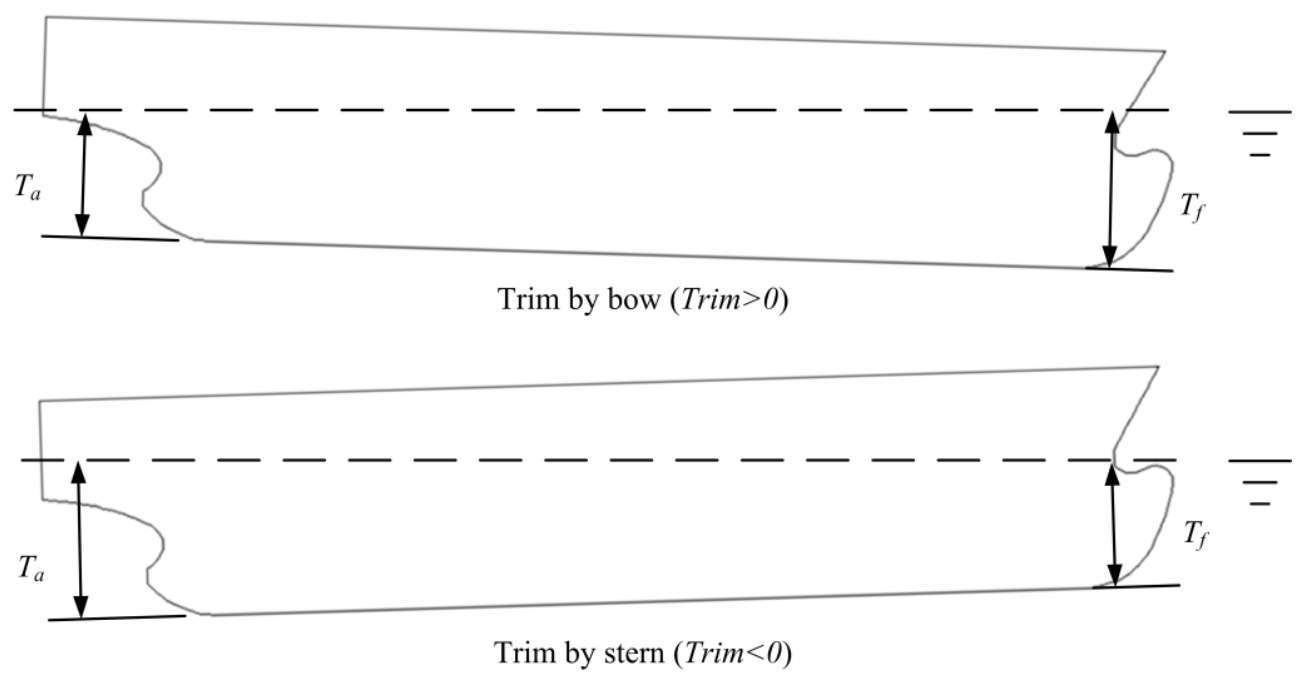

Fig. 7 Sketch of trim conditions for the hull 
Comparisons of wave resistance for the hull at two different trim conditions between numerical results and experiment results are shown in Fig. 8. The variation trend of calculated and experimental wave resistance with respect to trim conditions agrees quite well at a wide range of Froude number (i.e. Fn $>0.17$ ), even at the inflection point (i.e. Fn $\approx 0.192$, Fn $\approx$ 0.207) where the trim variations leads to few changes in wave resistance. During the experiment, the HUST hull is tested with free sinkage and it's found that sinkage of the hull is more significant at $\mathrm{Fn}=0.203$ than that at other Froude numbers. Since the wave resistance calculation was conducted with fixed sinkage for the HUST hull, one possible reason for the peak difference between wave resistance curves from calculation and experiment $(\mathrm{Fn}=0.203$ in Fig. 8) is that, the fixed sinkage during calculation covers up the influence of significant free sinkage to wave resistance. Typical wave profiles for the hull at $\mathrm{Fn}=0.203$ and 0.217 are shown in Figs 9 and 10 respectively. The variation trend of wave heights over trims from calculation shows a good agreement with that from experiment as well. In addition, calculated wave patterns provide further verification by detailing wave height changes with different trim conditions around the hull, as depicted in Figs 11 and 12. Thus, it is can be concluded that the present numerical procedure is able to properly demonstrate the wave resistance changes caused by trim adjustment for the HUST hull at Fn $>0.17$.

\section{Trim optimization}

To explore the relationship between a few explanatory variables (i.e. trim condition, Froude number) and a response variable (i.e. wave resistance), the response surface methodology is introduced to conduct trim optimization $[16,22]$. Instead of using a seconddegree polynomial model to approximate the surface, the Lagrange interpolation function is applied to make sure that the effective points from calculation is included in the response sutrface.

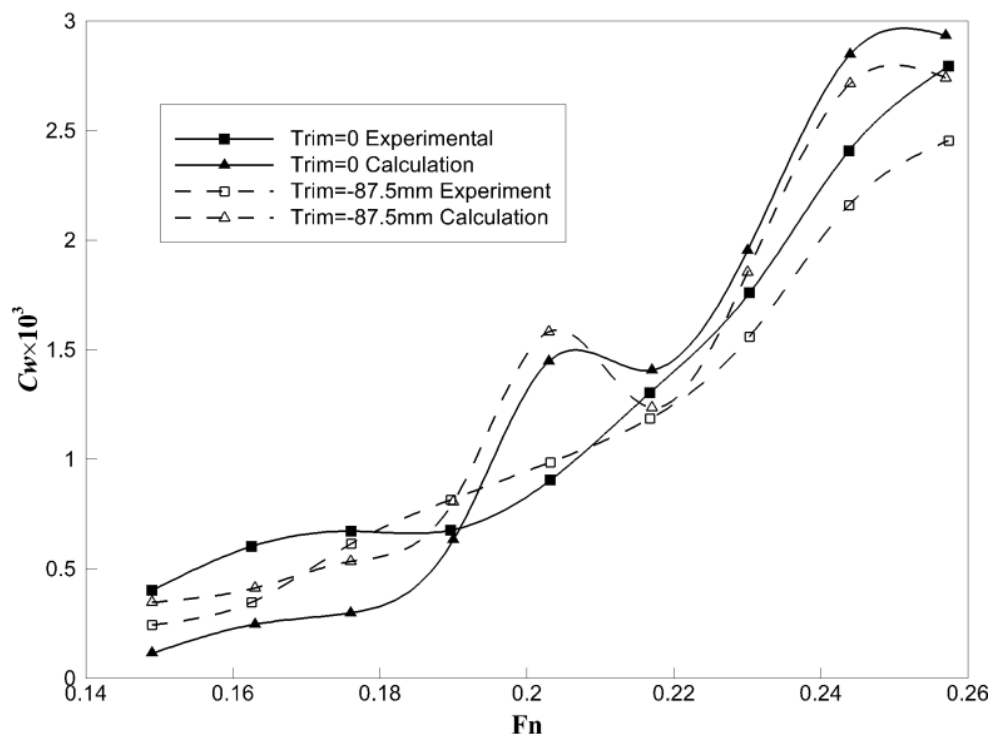

Fig. 8 Wave resistance of the HUST hull 


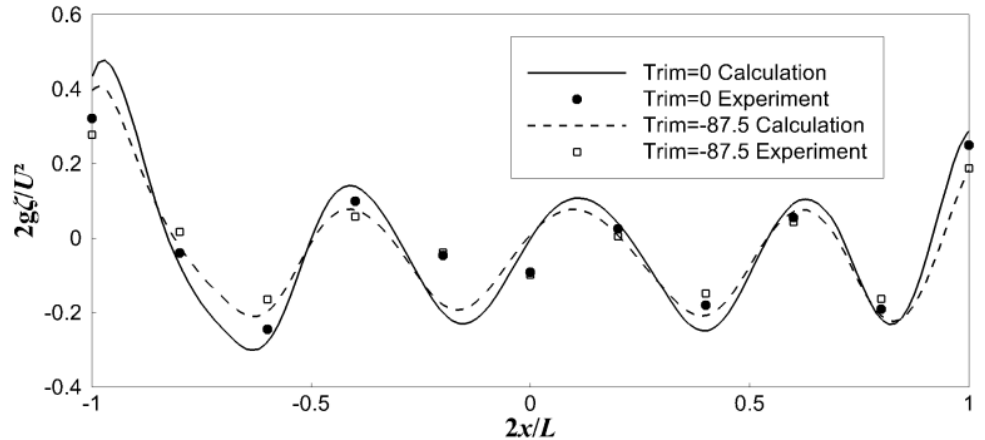

Fig. 9 Wave profiles along the HUST hull at $F n=0.203$

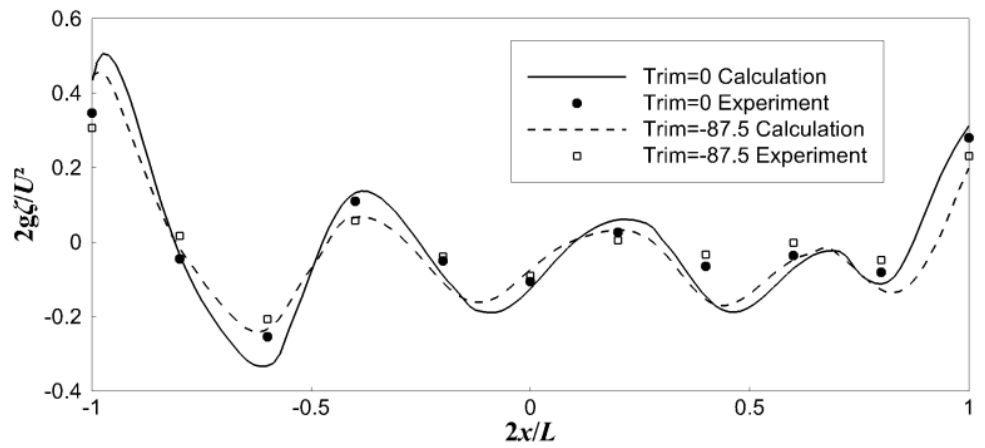

Fig. 10 Wave profiles along the HUST hull at Fn=0.217

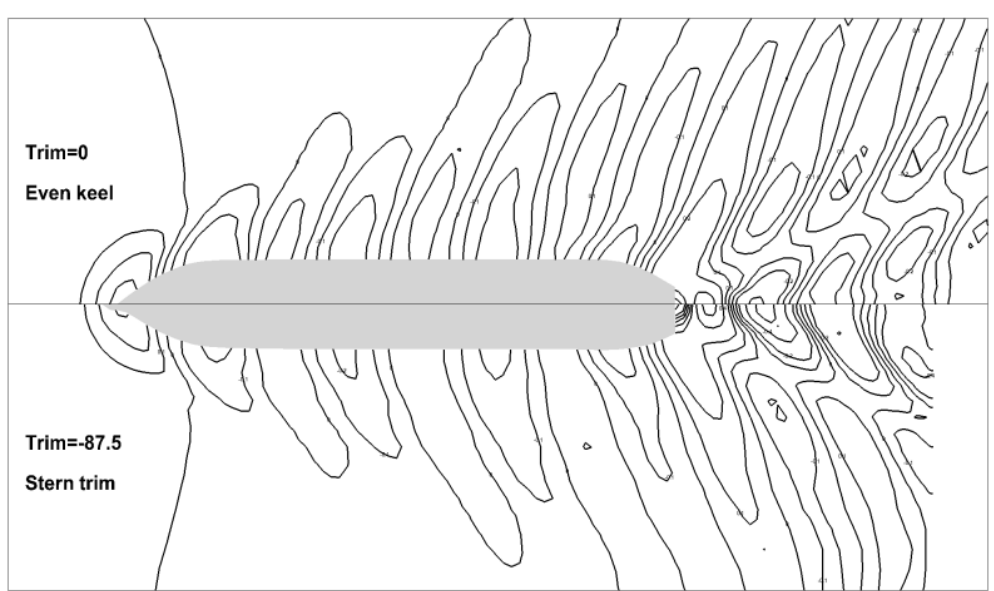

Fig. 11 Wave patterns around the HUST hull at Fn=0.203

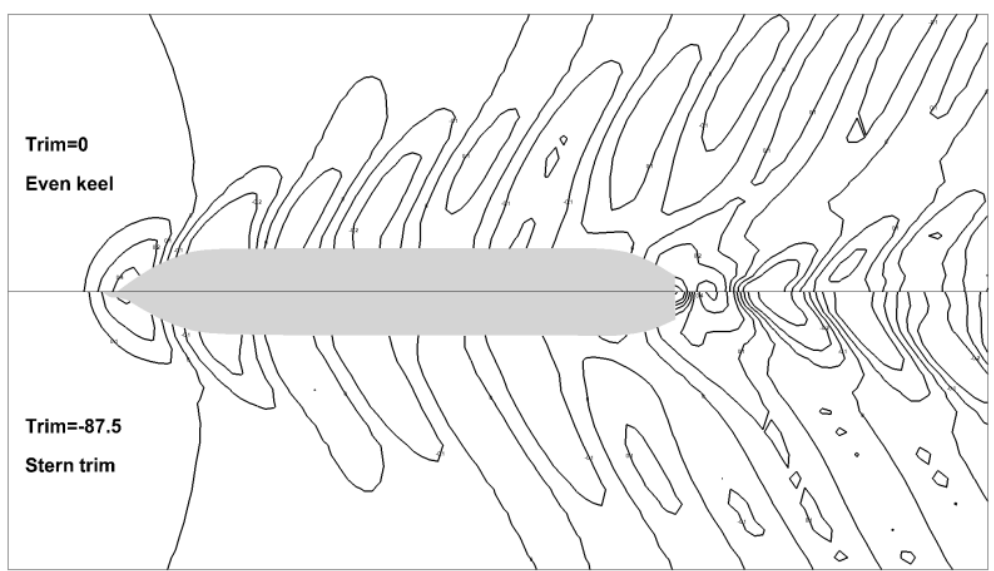

Fig. 12 Wave patterns around the HUST hull at Fn=0.217 
To create a database, the wave resistance of the HUST hull at different Froude numbers and trims is calculated with above numerical method and summarized in Table 2. The response surface of wave resistance with respect to Froude numbers and trims is constructed based on the wave resistance database, as depicted in Fig. 13.

The trim optimization of the HUST hull is conducted by using the response surface, in which a response of wave resistance is influenced by trim and Froude number. In present trim optimization for the HUST hull with the displacement at a certain initial draft (i.e. $D=268.7$ $\mathrm{mm}$ ), the design variables are trim and ship speed based Froude number. The objective is to find the optimal value of trim to minimize the wave resistance of the hull at a given Froude number. The optimization model is given as follows:

$$
\begin{aligned}
& C_{w}=f(\text { Trim }, \text { Fn, Dis }) \\
& \begin{cases}\text { min } & f(\text { Dis, Fn, Trim }) \\
\text { st. } & \left\{\begin{array}{l}
\text { Dis }=\text { Dis } 0 \\
\text { Fn }=\text { Fn } 0 \\
\text { Trim } 1<\text { Trim }<\text { Trim } 2
\end{array}\right.\end{cases}
\end{aligned}
$$

where $f($ Trim, Fn, Diss) is the response surface, DisO is present volume displacement of the hull, FnO is the Froude number based on given speed, and Triml and Trim 2 define the variation range of the trim condition. The overall optimization procedure is demonstrated in Fig. 14. There exists an optimal trim curve (may be made up by several curve portions) on the response surface, and the corresponding wave resistance is the minimum. The intersection point of the optimal trim curve and the Froude number isoline represents the optimal point of the minimum wave resistance at present ship velocity, as shown in Fig. 13 and detailed in Figs 15-17.

To evaluate the trim optimization results, the wave resistance reduction ratio is defined as

$$
\alpha_{w}=\frac{\left(C_{w \text { Optimum }}-C_{w}\right)}{C_{w}} \cdot 100 \%
$$

and the total resistance reduction ratio is defined as

$$
\alpha_{t}=\frac{\left(C_{t \text { Optimum }}-C_{t}\right)}{C_{t}} \cdot 100 \%
$$

where $C_{w O p t i m u m}$ and $C_{t \text { Optimum }}$ are wave resistance coefficient and total resistance coefficient at the optimum trim condition, respectively. $C_{w}$ and $C_{t}$ are wave resistance coefficient and total resistance coefficient at the reference trim condition point, respectively. The optimized trim condition is able to provide $9.7 \%-11.5 \%$ of wave resistance reduction and $3.5 \%-3.8 \%$ of total resistance reduction, compared with the even keel condition for the HUST hull. What's more,

Table 2 Numerical results of wave resistance for the HUST hull

\begin{tabular}{|c|c|c|c|c|c|c|c|c|c|}
\hline \multicolumn{9}{|c|}{$C_{w} \times 10^{3}$} \\
\hline Tirm $(\mathrm{mm})$ & 0.149 & 0.163 & 0.176 & 0.190 & 0.203 & 0.217 & 0.230 & 0.244 & 0.257 \\
\hline-87.5 & 0.346 & 0.221 & 0.559 & 0.755 & 1.399 & 1.267 & 1.980 & 2.951 & 3.016 \\
\hline-74.0 & 0.251 & 0.166 & 0.834 & 0.785 & 1.330 & 1.618 & 2.460 & 3.258 & 3.097 \\
\hline-59.0 & 0.208 & 0.239 & 0.719 & 0.727 & 1.288 & 1.474 & 2.265 & 3.126 & 2.981 \\
\hline
\end{tabular}




\begin{tabular}{|c|c|c|c|c|c|c|c|c|c|}
\hline-44.0 & 0.198 & 0.354 & 0.446 & 0.645 & 1.284 & 1.162 & 1.823 & 2.823 & 2.810 \\
\hline-29.0 & 0.171 & 0.377 & 0.255 & 0.592 & 1.315 & 1.041 & 1.599 & 2.666 & 2.756 \\
\hline-14.0 & 0.117 & 0.282 & 0.239 & 0.592 & 1.375 & 1.217 & 1.745 & 2.756 & 2.859 \\
\hline 0 & 0.114 & 0.246 & 0.298 & 0.631 & 1.447 & 1.406 & 1.951 & 2.848 & 2.933 \\
\hline 14.0 & 0.178 & 0.319 & 0.326 & 0.686 & 1.523 & 1.435 & 2.004 & 2.812 & 2.896 \\
\hline 30.0 & 0.217 & 0.307 & 0.268 & 0.731 & 1.588 & 1.369 & 1.944 & 2.781 & 2.871 \\
\hline 45.0 & 0.161 & 0.235 & 0.273 & 0.758 & 1.606 & 1.300 & 1.877 & 2.800 & 2.874 \\
\hline 56.0 & 0.103 & 0.412 & 0.533 & 0.805 & 1.582 & 1.235 & 1.853 & 2.714 & 2.740 \\
\hline
\end{tabular}

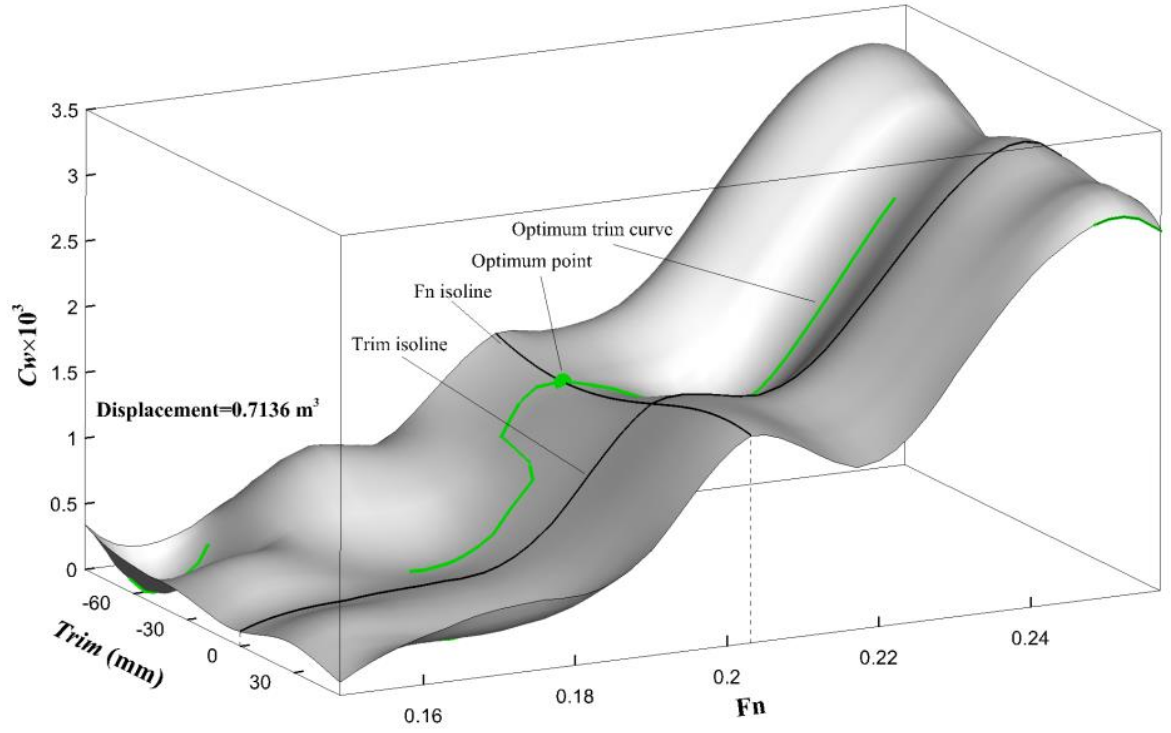

Fig. 13 Response surface of wave resistance

Optimization problem setup

Design variables, Objective functions, Constraints

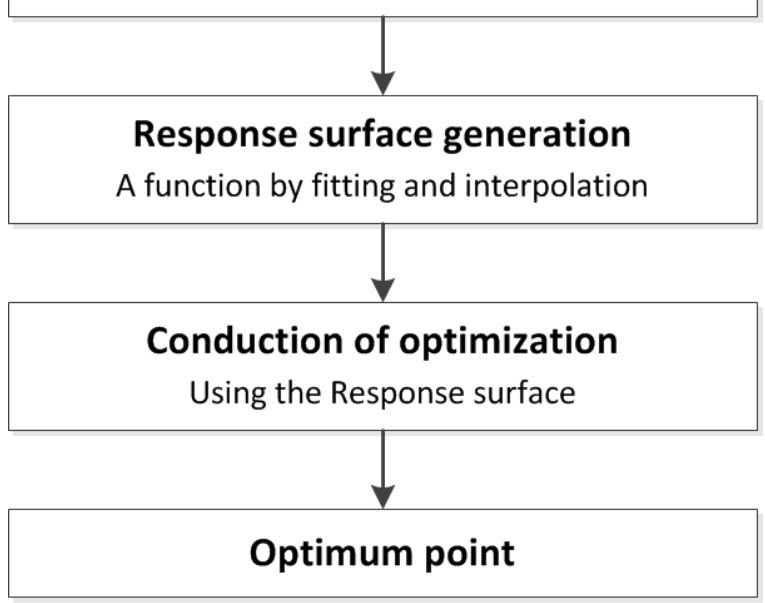

Fig. 14 Optimization procedure based on response surface 


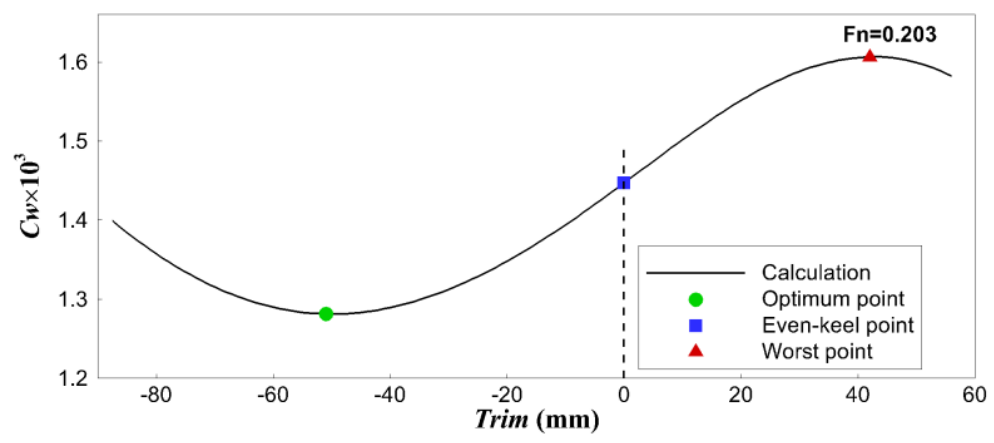

Fig. 15 Froude number isoline of response surface at $\mathrm{Fn}=0.203$

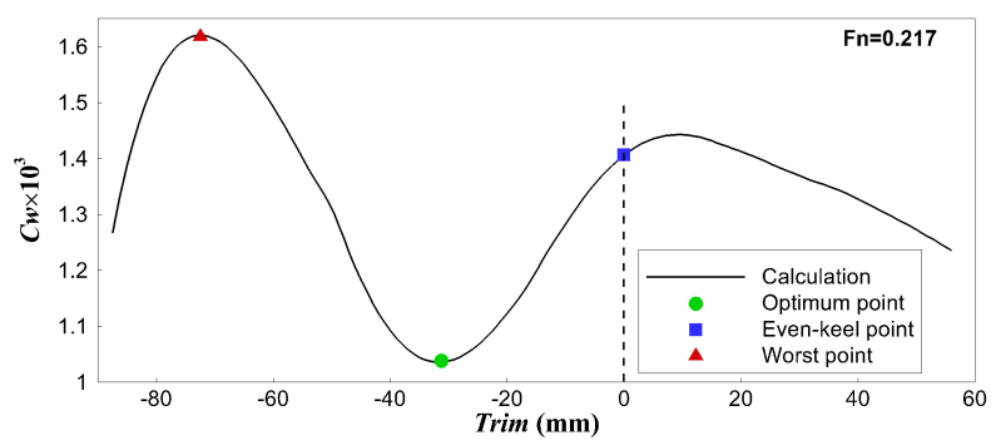

Fig. 16 Froude number isoline of response surface at $\mathrm{Fn}=0.217$

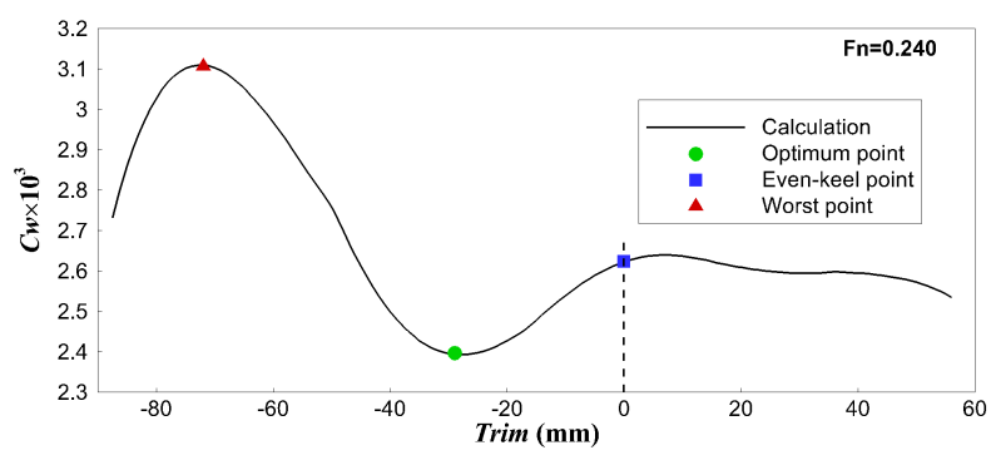

Fig. 17 Froude number isoline of response surface at $\mathrm{Fn}=0.240$

Table 3 Resistance reduction ratio at optimum trim condition point

\begin{tabular}{|l|c|c|c|}
\hline Fn & 0.203 & 0.217 & 0.240 \\
\hline$C_{w \text { Optimum }} \times 10^{3}$ & 1.281 & 1.038 & 2.397 \\
\hline$\alpha_{w}$ related to Even-keel point & $11.5 \%$ & $26.2 \%$ & $9.7 \%$ \\
\hline$\alpha_{w}$ related to Worst point & $20.2 \%$ & $35.8 \%$ & $23.7 \%$ \\
\hline$\alpha_{t}$ related to Even-keel point & $3.5 \%$ & $7.2 \%$ & $3.8 \%$ \\
\hline$\alpha_{t}$ related to Worst point & $6.8 \%$ & $11.3 \%$ & $11.8 \%$ \\
\hline
\end{tabular}

the reduction ratio can be as high as $20.2 \%-35.8 \%$ of wave resistance and $6.8 \%-11.3$ of total resistance while the comparison is taken between the optimized trim condition and the worst trim condition, as shown in Table 3. Obviously, the contribution of trim optimization to resistance reduction for the HUST hull is significant. 


\section{Conclusion}

Traditionally, hull lines are designed or optimized for minimum resistance at the designing trim condition, which is the even keel trim condition or trimming somewhat aft for most ships. To achieve reduction of resistance in a different way, the paper in which typical wave making of a hull is taken into account therefore aims at exploring a trim for the HUST hull, with minimum wave resistance at given Froude numbers and displacement. After being validated by results from calculation of the S60 hull and resistance test of the HUST hull, a potential-based panel method coupled with a response surface method is used to develop a framework for trim optimization. The optimum trim condition for the hull shows a significant improvement in both wave resistance and total resistance. The optimization framework is effective in energy saving due to resistance reduction, not only for the present HUST hull, but also for general hull models.

Acknowledgement: The authors gratefully acknowledge the financial support from the National Natural Science Foundation of China (NSFC) under Project Number 51609115 \& 51679097, and the financial support from the Opening Fund of the Hubei Key Laboratory of Naval Architecture \& Ocean Engineering Hydrodynamics under Project Number 201701 as well.

\section{REFERENCES}

[1] Traut, M., Gilbert, P., Walsh, C., Bows, A., Filippone, A., Stansby, P., Wood, R.: Propulsive power contribution of a kite and a Flettner rotor on selected shipping routes, Applied Energy, 113: 362-372, 2014. https://doi.org/10.1016/j.apenergy.2013.07.026

[2] Gibbs, D., Rigot-Muller, P., Mangan, J., Lalwani, C.: The role of sea ports in end-to-end maritime transport chain emissions, Energy Policy, 64: 337-348, 2014. https://doi.org/10.1016/j.enpol.2013.09.024

[3] Huang, F., Wang, L., Yang, C.: A new improved artificial bee colony algorithm for ship hull form optimization, Engineering Optimization, DOI: 10.1080/0305215X.2015.1031660, 2015. https://doi.org/10.1080/0305215X.2015.1031660

[4] Ayob, A. F., Nik, W. W., Ray, T., Smith, W. F.: Hull surface information retrieval and optimization of high speed planing craft, IOP Conference Series: Materials Science and Engineering, 2012. https://doi.org/10.1088/1757-899X/36/1/012034

[5] Tahara, Y., Peri, D., Campana, E. F., Stern, F.: Single- and multiobjective design optimization of a fast multihull ship: numerical and experimental results, Journal of Marine Science and Technology, 16(4): 412-433, 2011. https://doi.org/10.1007/s00773-011-0137-y

[6] Sun, J., Lv, X., Liu, W., Ning, H., Chen, X.: Research on a method of hull form design based on wavemaking resistance optimization, Polish Maritime Research, 19(3): 16-25, 2012.

[7] Banks, C., Turan, O., Incecik, A., Theotokatos, G., Izkan, S., Shewell, C., Tian, X.: Understanding ship operating profiles with an aim to improve energy efficient ship operations, Proceedings of the Low Carbon Shipping Conference, London, 2013.

[8] Lee, C., Newman, J. N.: Computation of wave effects using the panel method, Numerical Models in Fluid Structure Interaction, 42: 211-251, 2005. https://doi.org/10.2495/978-1-85312-837-0/06

[9] Yan, H., Liu, Y.: An efficient high-order boundary element method for nonlinear wave-wave and wave-body interactions, Journal of Computational Physics, 230(2): 402-424, 2011.

https://doi.org/10.1016/j.jcp.2010.09.029

[10] Belibassakis, K. A., Gerostathis, T. P., Kostas, K. V., Politis, C. G., Kaklis, P. D., Ginnis, A. I., Feurer, C.: A BEM-isogeometric method for the ship wave-resistance problem, Ocean Engineering, 60: 53-67, 2013. https://doi.org/10.1016/j.oceaneng.2012.12.030

[11] Liu, S. K., Papanikolaou, A., Zaraphonitis, G.: Prediction of added resistance of ships in waves, Ocean Engineering, 38(4): 641-650, 2011. https://doi.org/10.1016/j.oceaneng.2010.12.007

[12] Ghassemi, H., Kohansal, A. R.: Wave generated by the NACA 4412 hydrofoil near free surface, Journal of Applied Fluid Mechanics, 6(1): 1-6, 2013. 
[13] Peng, H., Ni, S., Qiu, W.: Wave pattern and resistance prediction for ships of full form, Ocean Engineering, 87: 162-173, 2014. https://doi.org/10.1016/j.oceaneng.2014.06.004

[14] Iakovatos, M. N., L Iarokapis, D. E., Andtzabiras, G. D.: Experimental investigation of the trim influence on the resistance characteristics of five ship models, Development in Marine Transportation and Exploitation of Sea Resources, 1: 23-32, 2014

[15] Sherbaz, S., Duan, W.: Ship Trim Optimization: Assessment of Influence of Trim on Resistance of MOERI Container Ship, The Scientific World Journal, 2014, 2014.

[16] Sun, J., Tu, H., Chen, Y., Xie, D., Zhou, J.: A study on trim optimization for a container ship based on effects due to resistance, Journal of Ship Research, 60(1): 30-47, 2016. https://doi.org/10.5957/JOSR.60.1.150022

[17] Lv, X., Wu, X., Sun, J., Tu, H.: Trim optimization of ship by a potential-based panel method, Advances in Mechanical Engineering, 5: 378140, 2013. https://doi.org/10.1155/2013/378140

[18] Tarafder, M. S., Suzuki, K.: Wave-making resistance of a catamaran hull in shallow water using a potentialbased panel method, Journal of Ship Research, 52(1): 16-29, 2008.

[19] Morino, L., Chen, L. T., Suciu, E. O.: Steady and oscillatory subsonic and supersonic aerodynamics around complex configurations, AIAA Journal, 13(3): 368-374, 1974.

[20] Tarafder, M. S., Suzuki, K.: Computation of wave-making resistance of a catamaran in deep water using a potential-based panel method, Ocean Engineering, 34(13): 1892-1900, 2007.

https://doi.org/10.1016/j.oceaneng.2006.06.010

[21] Toda, Y., Stern, F., Longo, J.: Mean-flow measurements in the boundary layer and wake and wave field of a Series $60 \mathrm{Cb}=0.6$ ship model - part 1: Froude numbers 0.16 and 0.316, Journal of ship Research, 36(4): 360-377, 1992.

Submitted: $\quad$ 10.03.2017. Xujian Lyu ${ }^{\mathbf{1 , 3}}$

Haiwen $\mathrm{Tu}^{2}$

Accepted: $\quad$ 18.09.2017. $\quad$ De Xie ${ }^{\mathbf{2 , 3}, \mathbf{4}}$,

Jianglong Sun 2, 3, 4, *

${ }^{1}$ School of Energy and Power Engineering, Nanjing University of Science and

Technology, Nanjing 210094, P. R. China

${ }^{2}$ School of Naval Architecture and Ocean Engineering, Huazhong University of Science and Technology, Hubei, P. R. China

${ }^{3}$ Hubei Key Laboratory of Naval Architecture and Ocean Engineering Hydrodynamics, Huazhong University of Science and Technology, Hubei, P. R. China

${ }^{4}$ Collaborative Innovation Center for Advanced Ship and Deep-Sea Exploration, Shanghai, P. R. China

* Corresponding author (J. Sun): sundapao@ 126.com (Email); +86-27- 87542946 (Fax); +86-27- 87543358 (Tel) 